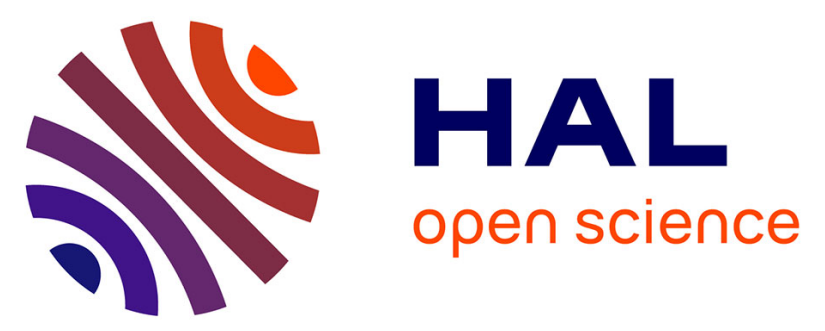

\title{
Synergistic antiarrhythmic effect of inward rectifier current inhibition and pulmonary vein isolation in a 3D computer model for atrial fibrillation
}

\author{
Ali Gharaviri, Simone Pezzuto, Mark Potse, Giulio Conte, Stef Zeemering, \\ Vladimír Sobota, Sander Verheule, Rolf Krause, Angelo Auricchio, Ulrich \\ Schotten
}

\section{To cite this version:}

Ali Gharaviri, Simone Pezzuto, Mark Potse, Giulio Conte, Stef Zeemering, et al.. Synergistic antiarrhythmic effect of inward rectifier current inhibition and pulmonary vein isolation in a $3 \mathrm{D}$ computer model for atrial fibrillation. EP-Europace, 2021, 23 (Supplement_1), pp.i161-i168. 10.1093/europace/euaa413 . hal-03452233

\section{HAL Id: hal-03452233 \\ https://hal.inria.fr/hal-03452233}

Submitted on 26 Nov 2021

HAL is a multi-disciplinary open access archive for the deposit and dissemination of scientific research documents, whether they are published or not. The documents may come from teaching and research institutions in France or abroad, or from public or private research centers.
L'archive ouverte pluridisciplinaire HAL, est destinée au dépôt et à la diffusion de documents scientifiques de niveau recherche, publiés ou non, émanant des établissements d'enseignement et de recherche français ou étrangers, des laboratoires publics ou privés. 
1 Synergistic antiarrhythmic effect of inward rectifier current 2 inhibition and pulmonary vein isolation in a 3D computer model 3 for atrial fibrillation

4 Ali Gharaviri, $\mathrm{PhD}^{\mathrm{a}}$, Simone Pezzuto ${ }^{\mathrm{a}}, \mathrm{PhD}$, Mark Potse, $\mathrm{PhD}^{\mathrm{b}, \mathrm{c}, \mathrm{d}}$, Giulio Conte, MD, $\mathrm{PhD}^{\mathrm{a}, \mathrm{e}}$,

5 Stef Zeemering, $\mathrm{PhD}^{\mathrm{f}}$, Vladimír Sobota, $\mathrm{MSc}^{\mathrm{f}}$, Sander Verheule, $\mathrm{PhD}^{\mathrm{f}}$, Rolf Krause, $\mathrm{PhD}^{\mathrm{a}}$,

6 Angelo Auricchio, MD, $\mathrm{PhD}^{\mathrm{a}, \mathrm{e}}$, Ulrich Schotten, $\mathrm{MD}, \mathrm{PhD}^{\mathrm{f}}$

7 a: Center for Computational Medicine in Cardiology, Institute of Computational Science,

8 Università della Svizzera italiana, Lugano, Switzerland.

9 b: Carmen team, Inria Bordeaux - Sud-Ouest, Talence, France.

c: Université de Bordeaux, IMB, UMR 5251, F-33400 Talence, France.

d: IHU Liryc, Electrophysiology and Heart Modeling Institute, foundation Bordeaux

12 Université, Bordeaux, France.

e: Fondazione Cardiocentro Ticino, Lugano, Switzerland.

f: Department of Physiology, Maastricht University, Maastricht, The Netherlands.

\section{Corresponding author:}

Angelo Auricchio, MD PhD

Division of Cardiology, Fondazione Cardiocentro Ticino

Via Tesserete 48

6900 Lugano, Switzerland

Tel.: +41918053340

E-Mail: angelo.auricchio@cardiocentro.org

Number of words (excluding references, table, and figures): 3625 
Aims: Recent clinical studies showed that antiarrhythmic drug (AAD) treatment and pulmonary vein isolation (PVI) synergistically reduce atrial fibrillation $(\mathrm{AF})$ recurrences after initially successful ablation. Among newly developed atrial-selective AADs, inhibitors of the G-protein-gated acetylcholine-activated inward rectifier current ( IKACh $\left._{\text {KCh }}\right)$ were shown to effectively suppress AF in an experimental model but have not yet been evaluated clinically. We tested in-silico whether inhibition of inward rectifier current or its combination with PVI reduces AF inducibility.

Methods: We simulated the effect of inward rectifier current blockade (IK blockade), PVI, and their combination on AF inducibility in a detailed 3-dimensional model of the human atria with different degrees of fibrosis. $I_{K}$ blockade was simulated with a $30 \%$ reduction of its conductivity. AF was initiated using incremental pacing applied at 20 different locations, in both atria.

Results: $\mathrm{I}_{\mathrm{K}}$ blockade effectively prevented $\mathrm{AF}$ induction in simulations without fibrosis as did PVI in simulations without fibrosis and with moderate fibrosis. Both interventions lost their efficacy in severe fibrosis. The combination of $\mathrm{I}_{\mathrm{K}}$ blockade and PVI prevented AF in simulations without fibrosis, with moderate fibrosis, and even with severe fibrosis. The combined therapy strongly decreased the number of fibrillation waves, due to a synergistic reduction of wavefront generation rate while the wavefront lifespan remained unchanged. Conclusions: Newly developed blockers of atrial specific inward rectifier currents, such as IKAch, might prevent AF occurrences and when combined with PVI effectively supress AF recurrences in human.

Keywords: Antiarrhythmic drug, Inward rectifier currents, Catheter ablation, Atrial Fibrillation recurrence, In-silico study. 
- We simulated the efficacy of inward rectifier current blockade and its combination with PVI on prevention of AF initiation in different degrees of atrial fibrosis. size, and thereby strongly prevented AF induction. 


\section{Condensed Abstract}

61 Continuation of antiarrhythmic drugs after catheter ablation has been shown to reduces AF recurrences. We studied whether inhibition of inward rectifier current combined with catheter ablation reduces AF inducibility in a realistic 3D computer model of human atria. We showed that either inward rectifier current inhibition or PVI separately cannot significantly reduce AF initiation likelihood in the simulations with severe atrial fibrosis, while their combination can. 
Despite the development of pharmacological therapies for patients with atrial fibrillation (AF), a large proportion of AF patients are not arrhythmia-free. ${ }^{1}$ Pharmacological therapy is less effective in non-paroxysmal AF patients than in paroxysmal AF patients, indicating the significance of structural remodelling in the efficacy of pharmacological therapy. If the treatment with antiarrhythmic drugs (AAD) fails, patients receive catheter ablation (CA) as a second-line treatment. ${ }^{2} \mathrm{CA}$ is also associated with a rather disappointing long term outcome, as after one year AF reoccurs in up to $30 \%$ of patients after PVI, despite proven complete PV isolation supporting the presence of driver mechanisms of AF located outside the PV area. ${ }^{3,4}$ The recent EAST trial stresses the need for early and effective rhythm control strategies ${ }^{5}$ and combination of AADs with CA may be a strategy to achieve this. In a recent study, Duytschaever et al. demonstrated that continuing previously ineffective treatment with AAD after $\mathrm{CA}$ is associated with lower prevalence of atrial tachyarrhythmias after one year. ${ }^{6}$ However, the mechanisms underlying this synergistic inhibition of AF recurrences is not well understood. Also, current AADs having potential side effects such as ventricular proarrhythmia. For these reasons, atrial-selective AADs that do not affect ventricular conduction or repolarization have been developed. Examples are blockers of the ultra-rapid inward rectifier current ( $\mathrm{I}_{\mathrm{Kur}}$ ), small-conductance $\mathrm{Ca}^{2+}$-activated $\mathrm{K}^{+}$-currents (SK), and the G-proteingated acetylcholine-activated inward rectifier current (IKAch). A blocker of $I_{K A c h}(X A F-1407)$ has recently been demonstrated to effectively suppress $\mathrm{AF}$ in an experimental equine model. ${ }^{7}$ Here, we tested whether inward rectifier current blockade reduces AF inducibility and whether the combination of inward rectifier blockade with PVI prevents AF recurrences in a computer model of human atria. 


\section{Methods}

\subsection{Computational model of AF}

Computer simulations were performed on a highly detailed 3D model of human atria. ${ }^{8}$ The model geometry was based on magnetic resonance imaging data of a subject with normal atria with manually added anatomical structures and properties such as three layers of fibre orientations, endocardial trabeculated network, Bachmann's bundle, wall thickness heterogeneities, and left atrial appendage trabeculated network. ${ }^{8}$ Several studies have shown that these structures have an essential role in AF initiation and maintenance. ${ }^{9,10}$ The model consisted of approximately 5 million nodes spaced at $200 \mu \mathrm{m}$.

Action potential propagation was simulated with a monodomain reaction-diffusion equation using the Courtemanche-Ramirez-Nattel atrial cell model. ${ }^{11}$ AF-induced changes in ionic currents were incorporated by setting the conductivities for $\mathrm{I}_{\mathrm{to}}, \mathrm{I}_{\mathrm{Ca}, \mathrm{L}}$, and $\mathrm{I}_{\mathrm{K} 1}$ at $40 \%, 35 \%$, and $200 \%$ of their normal values, respectively. ${ }^{12}$ Simulations were performed using the propag-5 software $^{13}$ and run on a Cray XC50 supercomputer with GPU support.

\subsection{Modelling of inward rectifier current blockade}

The inward rectifier background current $\left(\mathrm{I}_{\mathrm{K} 1}\right)$ and the G-protein-gated acetylcholine-activated inward rectifier current $\left(\mathrm{I}_{\mathrm{KAch}}\right)$ show identical current-voltage relationships and are, therefore, indistinguishable on the single cell level. ${ }^{14}$ Thus, both currents are adequately represented by one total inward rectifier conductance in computer models. For this reason, inhibition of $\mathrm{I}_{\mathrm{KAch}}$ was simulated by reduction of this total inward rectifier current. The conductance of the current was reduced by $30 \%$ resulting in a prolongation of the atrial action potential duration by approximately $20 \mathrm{~ms}$, which is in the range of experimental studies. ${ }^{15,16}$ 


\subsection{Distribution of tissue fibrosis}

115 The effect of structural remodelling was simulated by different degrees of fibrosis. The

116 conditions without, with moderate, and with severe fibrosis were modelled by setting $0 \%$,

$11750 \%$, and $70 \%$ of the elements in the model as fibrotic, respectively. Fibrotic elements

118 remained conductive along the fibre direction and isolated in the two transverse directions.

119 This modelling of atrial tissue fibrosis agrees with the experimental study by Spach et al. that

120 revealed loss of side-to-side electrical connections between atrial muscle bundles in fibrotic

121 atrial tissue. ${ }^{17}$ The fibrosis was distributed within the subepicardial layer, while the atrial

122 bundles remained intact. The spatial distribution of fibrosis was uneven, with patches or

123 island presenting higher degree of fibrosis. Such distribution was based on spatially

124 correlated, anatomy-tailored random fields. ${ }^{8,18}$

\subsection{Modelling of Pulmonary vein isolation}

In PVI simulations, two antral circumferential lines isolated the PV from the atria. Virtual ablation lesions consisted of tissue volumes modelled by non-conductive elements.

\subsection{AF initiation in pre- and post-catheter ablations}

AF initiation likelihood was assessed by applying incremental pacing at 20 different locations in both atria and compared between the control (no inward rectifier current inhibition and no CA), inward rectifier current blockade ( $\mathrm{I}_{\mathrm{K}}$ blockade), PVI, and $\mathrm{I}_{\mathrm{K}}$ blockade + PVI groups. Pacing locations were selected based on reported possible sources of extra-PV ectopic focal activity in AF patients, including the area between the PVs, left atrium, left atrial appendage, right atrial appendage, coronary sinus, superior caval vein, inferior caval vein, and right atrium. ${ }^{19}$ All pacing sites were located outside the ablated area. In each simulation, AF was initiated by applying incremental pacing with a duration of 2 seconds at a selected pacing 
site. In total, 14 stimuli were applied with the pacing cycle length gradually reducing from $280 \mathrm{~ms}$ to $124 \mathrm{~ms}$. After the pacing period, the simulations were continued for another 3 seconds with no pacing.

\subsection{Definition of successful AF initiation}

The stimulation protocol outcome was analysed in terms of the type of self-sustained rhythm after 2 seconds of stimulation. If no activation after pacing was noted, the initiation was considered unsuccessful. Otherwise, a distinction between AF and atrial flutter (AFL) was made, with the latter not being considered as successful AF initiation. AF and AFL conduction patterns were differentiated by analysing the reconstructed 12-lead ECGs, as described in detail in the supplementary material.

\subsection{Detection and tracking of fibrillation waves}

A fibrillation wave was defined as a contiguous volume in which all nodes had transmembrane voltage above $-60 \mathrm{mV}$. Wave size was defined as the number of model elements within this contiguous volume. Both the number of waves and wave sizes were calculated at each millisecond of simulated time.

Fibrillation waves were tracked in both time and space, as described in our previous study. ${ }^{20}$ Briefly, the temporal dynamics of waves can be described by three events:

- Generation: appearance of a new wave either due to wave break or transmural conduction.

- Fusion: the merge of two or more waves into one wave.

- Extinction: the extinction of a wave, due to encountering either a boundary or unexcitable tissue. 


\subsection{Statistics}

160 All statistical analyses were performed using GraphPad Prism software version 8.0. The

161

values are expressed as means \pm SD. Statistical tests were performed to compare the effect of three parameters (fibrosis levels, $\mathrm{I}_{\mathrm{K}}$ blockade, and PVI) in AF initiation rate, using two-way ANOVA followed by post hoc Bonferroni test. A value of $\mathrm{P}<0.05$ was considered to be significant.

\section{Results}

In total, 240 simulations were performed. The simulations consisted of 4 groups: control (no interventions), inward rectifier current blockade ( $\mathrm{I}_{\mathrm{K}}$ blockade), PVI, and the combination of inward rectifier current blockade and PVI (IK blockade + PVI). Twenty simulations were performed for each degree of fibrosis, resulting in the total of 60 simulations performed in each group.

\subsection{AF initiation}

Figure 1A displays several action potentials recorded during simulated AF episodes in the control and $\mathrm{I}_{\mathrm{K}}$ blockade simulations. $\mathrm{I}_{\mathrm{K}}$ inhibition caused prolongation of action potential duration at $90 \%$ of repolarization $\left(\mathrm{APD}_{90}\right.$ ) from $119 \pm 12.5 \mathrm{~ms}$ in control to $136 \pm 10.4 \mathrm{~ms}$. Figure 2 depicts representative conduction patterns and corresponding ECG leads of $I_{K}$ blockade, PVI, and $\mathrm{I}_{\mathrm{K}}$ blockade + PVI simulations under the condition of severe fibrosis. As depicted in this figure, both $\mathrm{I}_{\mathrm{K}}$ blockade and PVI failed to prevent AF initiation in severe fibrosis simulations, while the combination rendered the atria uninducible.

Figure 3 shows initiation rates of atrial tachycardia in all simulation groups in the presence of different degrees of fibrosis. The figure shows that in the absence of fibrosis, $I_{K}$ blockade 
caused a significant reduction in AF initiation likelihood when compared to control, either by preventing initiation of $\mathrm{AF}$ or by transforming initiated $\mathrm{AF}$ into $\mathrm{AFL}$. $\mathrm{I}_{\mathrm{K}}$ blockade failed to significantly reduce AF initiation rate in the simulations with moderate or severe fibrosis. In PVI simulations, we observed significant reduction in AF initiation likelihood in the absence or in the presence of moderate fibrosis when compared to control, while no significant reduction was noted in the simulations with severe fibrosis. In the simulations that combined PVI with $\mathrm{I}_{\mathrm{K}}$ blockade, we observed a comparable reduction in $\mathrm{AF}$ initiation likelihood as in PVI simulations in the absence of fibrosis or in the presence of moderate fibrosis. The combination of $\mathrm{I}_{\mathrm{K}}$ blockade and PVI further reduced AF initiation likelihood by at least half in the simulations with severe fibrosis.

\subsection{Fibrillation pattern complexity}

Overall, a significant increase in $\mathrm{AF}$ cycle length was observed in all simulation groups when compared to control, regardless of the fibrosis degree, as shown in Table 1. When compared to control, no significant differences in the excitable gap were detected in any simulation group (see Table. 1).

Examples of wave lifespans in control, $\mathrm{I}_{\mathrm{K}}$ blockade, PVI, and PVI accompanied with $\mathrm{I}_{\mathrm{K}}$ blockade simulations with severe fibrosis are illustrated in figure 4A. An increase in the fibrosis degree in control simulations led to a significant increase in fibrillation wave generation rate, quantified as the slope of the fitted line to the wave lifespans, and therefore a significant increase in AF conduction pattern complexity, quantified as the average number of waves per cycle (Figure 4B \& C). The increase in conduction pattern complexity was due to the reduction of wave size and higher rate of new wavefront generation, while wavefront lifespan medians remained unchanged (Figure 4D \& E). In the simulations without fibrosis, $\mathrm{I}_{\mathrm{K}}$ blockade caused a significant reduction in the average number of waves per cycle, by 
increasing the wave sizes and significantly reducing the rate of wavefront generation. However, this effect of $I_{K}$ blockade disappeared in the simulations with moderate and severe fibrosis. PVI significantly reduced new wavefront generation rate in the simulations without fibrosis as well as in the simulations with moderate fibrosis. Nevertheless, this effect faded in PVI simulations with severe fibrosis. In IK blockade combined with PVI simulations, the synergistic interaction between the wave size enlargement caused by $\mathrm{I}_{\mathrm{K}}$ blockade and the reduction in atrial tissue mass caused by PVI further reduced the available space for wavefront interactions. This resulted in significantly decreased rate of new wavefront generation, and therefore in a smaller number of fibrillation waves.

\section{Discussion}

We investigated the isolated and combined effects of inward rectifier current blockade and PVI on AF initiation in the presence of various degrees of atrial fibrosis. Both inward rectifier blockade and PVI effectively reduced the rate of AF induction at low degrees of atrial fibrosis but lost their efficacy in severely fibrotic atria. In the simulations with severe fibrosis the combination of inward rectifier blockade and PVI effectively suppressed AF recurrences, while either of the interventions was not efficient individually. The synergy between both interventions resulted in reduction in the number of fibrillation waves. The main underlying mechanism behind this effect was a reduction in the rate of new wavefront generation, leaving the lifespan of fibrillation waves unaffected.

\subsection{Effect of inward rectifier current blockade and PVI in previous in-silico studies}

So far, several in-silico studies investigated the effect of different antiarrhythmic drugs (single-channel or multiple-channel block) or CAs on AF initiation, perpetuation and termination..$^{21,22}$ 
Among the large variety of potassium channels expressed in human atria, the inward rectifier currents $\mathrm{I}_{\mathrm{K} 1}$ and $\mathrm{I}_{\mathrm{KAch}}$ play a significant role in AF perpetuation. Heterogeneous shortening of the atrial effective refractory period caused by vagally modulated $\mathrm{I}_{\mathrm{KACh}}$ channels are thought to be the mechanism underlying vagally-induced $A F \cdot{ }^{23}$ Hence, inhibition of $\mathrm{I}_{\mathrm{KAch}}$ may minimize the effect of $I_{\mathrm{KACh}}$ modulations and could be a future therapeutic option for vagallyinduced AF. ${ }^{23}$ Dobrev and colleagues have demonstrated that an increase of constitutively active $\mathrm{I}_{\mathrm{KAch}}$ strongly contributes to atrial action potential shortening in human persistent $A F{ }^{14}$ Also $I_{K A c h}$ is active in atrial but not in ventricular cells so that inhibition of $I_{K A c h}$ is not expected to prolong ventricular action potential. For these reasons inward rectifier currents are an attractive target for antiarrhythmic treatment of AF. ${ }^{22,24}$ Importantly, $\mathrm{I}_{\mathrm{K} 1}$ and $\mathrm{I}_{\mathrm{KAch}}$ only differ in their single channel conductance but their single cell level current-voltage relationships are identical. ${ }^{14}$ Thus, both currents are adequately represented by the total inward rectifier conductance in computer models.

The influence of inward rectifier current inhibition on AF initiation, termination, and conduction pattern was investigated in several computational studies. ${ }^{21,22}$ Sánchez et al. demonstrated that inward rectifier inhibition led to higher arrhythmia organization by enlarging wave sizes, increasing wave meandering, and reducing the number of secondary wavelets. ${ }^{24}$ These findings are in line with our results of inward rectifier current inhibition in the atria without fibrosis in the present study. However, we observed significant efficacy loss of inward rectifier current blockade in reducing the number of fibrillation waves and $\mathrm{AF}$ termination or initiation in the presence of moderate or severe fibrosis. The effectiveness of PVI to terminate AF in representative virtual atria was assessed in several studies. ${ }^{25}$ Recent proof-of-concept in-silico studies investigated the role of atrial fibrosis in ablation failure using detailed patient-specific image-based models. ${ }^{26}$ These studies demonstrated that both the patient-specific degree and the distribution of fibrosis are a 
determining factor in $\mathrm{AF}$ initiation and maintenance. ${ }^{27}$ McDowell et al. demonstrated that modelling of ablation lesions in the persistent rotor core meandering regions renders $\mathrm{AF}$ uninducible. ${ }^{27}$ Here, we simulated the combination of PVI and inward rectifier current blockade and studied the mechanisms underlying the synergy between these two treatment options on prevention of AF initiation.

\subsection{Synergism between inward rectifier inhibition and PVI to prevent AF recurrence}

The development of efficient therapy against AF remains an important unfulfilled clinical need. Treatment with AADs has been considered as the first-line therapy ${ }^{2}$ and recently has been demonstrated to reduce major cardiovascular adverse events in patients with $\mathrm{AF} .{ }^{5}$ However, the efficacy of AADs decreases with AF progression. ${ }^{2} \mathrm{CA}$ has been recognized as an alternative therapeutic modality in $\mathrm{AF}$ treatment, which has been highlighted in several studies to be more efficient than AAD therapy to prevent AF recurrences. ${ }^{21}$ Nevertheless, CA results remain suboptimal, particularly in non-paroxysmal AF patients. ${ }^{28}$ Recently, there has been a growing interest in the effect of AAD continuation after CA. ${ }^{6}$ As reported in the POWDER-AF trial, the AADs, despite being inefficient before ablation, are becoming efficient in reducing AF recurrence after $\mathrm{CA} .{ }^{6}$ The mechanisms underlying this synergy between $\mathrm{AADs}$ and $\mathrm{CA}$ in $\mathrm{AF}$ recurrence prevention were so far not well understood. Our simulations showed that either inward rectifier current inhibition alone or PVI alone can significantly prevent initiation of AF only in the presence of no or moderate fibrosis, while both became inefficient in the presence of severe fibrosis. When both strategies were combined, little contribution to further reduction of AF inducibility in the simulations with no or moderate fibrosis was observed. In severe fibrosis, inward rectifier blockade in combination with PVI strongly reduced the number of fibrillation waves, increased their size and strongly prevented successful AF induction. This effect was associated with significant 
decline in the rate of new wave generation while wavefront lifespan remained unaltered. The observed synergism might be explained by the fact that both PVI as well as the wavelength prolongation by the inward rectifier blockade restrict the available space for wave-frontwave-tail interactions which reduces the likelihood for wave break and generation of new waves. Our results indicate that the newly developed inhibitors of $\mathrm{IKACh}_{\mathrm{K}}$ have a potential to show the observed synergism with PVI to prevent AF induction in a clinical setting.

\section{Limitations}

Inter-individual variabilities in anatomy, atrial size, fibre orientation, and wall thickness were not considered in our study. Several clinical and simulation studies reported the effect of variability in atrial geometry and the pattern of fibrosis on initiation of fibrillatory waves. ${ }^{26,}$ ${ }^{29-31}$ Studying the effect of variations in the pattern of atrial fibrosis on AF inducibility is certainly warranted. Although based on clinical data, the pattern of atrial fibrosis was generated algorithmically in this study. We investigated the effect of inward rectifier current inhibition, whereas several other currents have been shown to play an important role in AF termination. Moreover, we did not investigate inhibition of multiple ion channels on AF dynamics. Finally, heterogeneity in ionic parameters in our model has not been implemented on purpose in order to avoid confounding factors.

\section{Conclusion}

This study shows that adding inward rectifier current inhibition to PVI caused a significant reduction in $\mathrm{AF}$ recurrences even in the atria with severe fibrosis, whereas both treatments alone failed to prevent AF initiation in severely fibrotic atria. This reduction in AF recurrence rate is due to the synergistic effect of the two treatments to reduce the number of wavefrontwavetail interactions, thereby lowering the rate of new wavefront generation and thus the number of active fibrillation waves. 
374 This work was supported by the Swiss National Supercomputing Centre (CSCS) under project ID s778 and by grants to US from the Netherlands Heart Foundation (CVON2014-09,

RACE V: Reappraisal of Atrial Fibrillation: Interaction between hypercoagulability, Electrical remodelling, and Vascular Destabilisation in the Progression of AF), and the European Union (ERACoSysMED H2020 ERA-NET, Marie Sklodowska-Curie grant agreement No. 675351). Dr Conte is supported by the Swiss National Foundation (SNF) (Ambizione grant no PZ00P3_180055 / 1).

\section{Conflict of interest}

Angelo Auricchio (AA) is a consultant to Boston Scientific, Backbeat, Biosense Webster, Cairdac, Corvia, Microport CRM, Philips, Radcliffe Publisher. He received speaker fee from Boston Scientific, Medtronic, and Microport. He participates in clinical trials sponsored by Boston Scientific, Medtronic, Philips. He has intellectual properties with Boston Scientific, Biosense Webster, and Microport CRM. Ulrich Schotten (US) received consultancy fees or honoraria from Johnson \& Johnson, Roche Diagnostics, and Bayer Healthcare. US is cofounder and shareholder of YourRhythmics BV. He holds intellectual property with Roche and YourRhythmics BV. The other authors have nothing to declare. 


\section{Reference:}

[1] Rillig A, Lin T, Ouyang F, Heinz Kuck K, Richard Tilz R. Comparing Antiarrhythmic Drugs and Catheter Ablation for Treatment of Atrial Fibrillation. J Atr Fibrillation 2013; 6: 861.

[2] Hakalahti A, Biancari F, Nielsen JC, Raatikainen MJ. Radiofrequency ablation vs. antiarrhythmic drug therapy as first line treatment of symptomatic atrial fibrillation: systematic review and meta-analysis. Europace 2015; 17: 370-378.

[3] Vogler J, Willems S, Sultan A, Schreiber D, Luker J, Servatius H, et al. Pulmonary Vein Isolation Versus Defragmentation: The CHASE-AF Clinical Trial. J Am Coll Cardiol 2015; 66: 2743-2752.

[4] Kuck KH, Brugada J, Furnkranz A, Metzner A, Ouyang F, Chun KR, et al. Cryoballoon or Radiofrequency Ablation for Paroxysmal Atrial Fibrillation. N Engl J Med 2016; 374: 2235-2245.

[5] Kirchhof P, Camm AJ, Goette A, Brandes A, Eckardt L, Elvan A, et al. Early Rhythm-Control Therapy in Patients with Atrial Fibrillation. N Engl J Med 2020.

[6] Duytschaever M, Demolder A, Phlips T, Sarkozy A, El Haddad M, Taghji P, et al. PulmOnary vein isolation With vs. without continued antiarrhythmic Drug trEatment in subjects with Recurrent Atrial Fibrillation (POWDER AF): results from a multicentre randomized trial. Eur Heart J 2018; 39: 1429-1437.

[7] Fenner MF, Carstensen H, Dalgas Nissen S, Melis Hesselkilde E, Scott Lunddahl C, Adler Hess Jensen M, et al. Effect of selective IK,ACh inhibition by XAF-1407 in an equine model of tachypacing-induced persistent atrial fibrillation. Br J Pharmacol 2020; 177: 37783794.

[8] Gharaviri A, Bidar E, Potse M, Zeemering S, Verheule S, Pezzuto S, et al. Epicardial Fibrosis Explains Increased Endo-Epicardial Dissociation and Epicardial Breakthroughs in Human Atrial Fibrillation. Front Physiol 2020.

[9] Whitaker J, Rajani R, Chubb H, Gabrawi M, Varela M, Wright M, et al. The role of myocardial wall thickness in atrial arrhythmogenesis. Europace 2016; 18: 1758-1772.

[10] Verheule S, Eckstein J, Linz D, Maesen B, Bidar E, Gharaviri A, et al. Role of endoepicardial dissociation of electrical activity and transmural conduction in the development of persistent atrial fibrillation. Prog Biophys Mol Biol 2014; 115: 173-185.

[11] Courtemanche M, Ramirez RJ, Nattel S. Ionic mechanisms underlying human atrial action potential properties: insights from a mathematical model. Am J Physiol 1998; 275: H301-321.

[12] Gharaviri A, Verheule S, Eckstein J, Potse M, Kuklik P, Kuijpers NH, et al. How disruption of endo-epicardial electrical connections enhances endo-epicardial conduction during atrial fibrillation. Europace 2017; 19: 308-318.

[13] Krause D, Potse M, Dickopf T, Krause R, Auricchio A, Prinzen F. Hybrid Parallelization of a Large-Scale Heart Model. In: Keller R, Kramer D and Weiss J-P, eds. Facing the Multicore - Challenge II. Springer Berlin Heidelberg 2012: 120-132.

[14] Dobrev D, Friedrich A, Voigt N, Jost N, Wettwer E, Christ T, et al. The G proteingated potassium current $\mathrm{I}(\mathrm{K}, \mathrm{ACh})$ is constitutively active in patients with chronic atrial fibrillation. Circulation 2005; 112: 3697-3706.

[15] Dobrev D, Graf E, Wettwer E, Himmel HM, Hala O, Doerfel C, et al. Molecular basis of downregulation of G-protein-coupled inward rectifying $\mathrm{K}(+)$ current $(\mathrm{I}(\mathrm{K}, \mathrm{ACh})$ in chronic human atrial fibrillation: decrease in GIRK4 mRNA correlates with reduced I(K,ACh) and muscarinic receptor-mediated shortening of action potentials. Circulation 2001; 104: 25512557. 
[16] Voigt N, Abu-Taha I, Heijman J, Dobrev D. Constitutive activity of the acetylcholineactivated potassium current IK,ACh in cardiomyocytes. Adv Pharmacol 2014; 70: 393-409. [17] Spach MS, Dolber PC. Relating extracellular potentials and their derivatives to anisotropic propagation at a microscopic level in human cardiac muscle. Evidence for electrical uncoupling of side-to-side fiber connections with increasing age. Circ Res 1986; 58: $356-371$.

[18] Pezzuto S, Gharaviri A, Schotten U, Potse M, Conte G, Caputo ML, et al. Beat-tobeat $\mathrm{P}$-wave morphological variability in patients with paroxysmal atrial fibrillation: an in silico study. Europace 2018; 20: iii26-iii35.

[19] Santangeli P, Marchlinski FE. Techniques for the provocation, localization, and ablation of non-pulmonary vein triggers for atrial fibrillation. Heart Rhythm 2017; 14: 10871096.

[20] Gharaviri A, Verheule S, Eckstein J, Potse M, Kuijpers NH, Schotten U. A computer model of endo-epicardial electrical dissociation and transmural conduction during atrial fibrillation. Europace 2012; 14 Suppl 5: v10-v16.

[21] Grandi E, Dobrev D, Heijman J. Computational modeling: What does it tell us about atrial fibrillation therapy? Int J Cardiol 2019; 287: 155-161.

[22] Grandi E, Ripplinger CM. Antiarrhythmic mechanisms of beta blocker therapy. Pharmacol Res 2019; 146: 104274.

[23] Rattanawong P, Kewcharoen J, K SS, Shen WK. Drug Therapy for Vagally-Mediated Atrial Fibrillation and Sympatho-Vagal Balance in the Genesis of Atrial Fibrillation: A Review of the Current Literature. J Atr Fibrillation 2020; 13: 2410.

[24] Sanchez C, Bueno-Orovio A, Pueyo E, Rodriguez B. Atrial Fibrillation Dynamics and Ionic Block Effects in Six Heterogeneous Human 3D Virtual Atria with Distinct Repolarization Dynamics. Front Bioeng Biotechnol 2017; 5: 29.

[25] Rotter M, Dang L, Jacquemet V, Virag N, Kappenberger L, Haissaguerre M. Impact of varying ablation patterns in a simulation model of persistent atrial fibrillation. Pacing Clin Electrophysiol 2007; 30: 314-321.

[26] Grandi E, Maleckar MM. Anti-arrhythmic strategies for atrial fibrillation: The role of computational modeling in discovery, development, and optimization. Pharmacol Ther 2016; 168: $126-142$.

[27] McDowell KS, Zahid S, Vadakkumpadan F, Blauer J, MacLeod RS, Trayanova NA. Virtual electrophysiological study of atrial fibrillation in fibrotic remodeling. PLoS One 2015; 10: e0117110.

[28] Verma A, Jiang CY, Betts TR, Chen J, Deisenhofer I, Mantovan R, et al. Approaches to catheter ablation for persistent atrial fibrillation. $N$ Engl J Med 2015; 372: 1812-1822.

[29] Boyle PM, Zahid S, Trayanova NA. Using personalized computer models to customtailor ablation procedures for atrial fibrillation patients: are we there yet? Expert Rev Cardiovasc Ther 2017; 15: 339-341.

[30] Zahid S, Whyte KN, Schwarz EL, Blake RC, 3rd, Boyle PM, Chrispin J, et al. Feasibility of using patient-specific models and the "minimum cut" algorithm to predict optimal ablation targets for left atrial flutter. Heart Rhythm 2016; 13: 1687-1698.

[31] Ali RL, Hakim JB, Boyle PM, Zahid S, Sivasambu B, Marine JE, et al. Arrhythmogenic propensity of the fibrotic substrate after atrial fibrillation ablation: a longitudinal study using magnetic resonance imaging-based atrial models. Cardiovasc Res 2019; 115: 1757-1765. 
Figure 1: A) Simulated action potentials during AF episodes in control and $\mathrm{I}_{\mathrm{K}}$ blockade simulations. Examples of simulated ECG in B) control and C) $I_{K}$ blockade simulation. Figure 2: Consecutive snapshots of conduction patterns and corresponding ECG leads (II, V1, and V3) in $I_{K}$ blockade, PVI, and $I_{K}$ blockade combined with PVI simulations with severe fibrosis. Black star indicates the stimulation point.

Figure 3: Atrial fibrillation (AF) and atrial flutter (AFL) initiation likelihood in control (no $\mathrm{I}_{\mathrm{K}}$ blockade and no PVI), PVI, and PVI $+\mathrm{I}_{\mathrm{K}}$ blockade with different degrees of fibrosis. Figure 4: Electrophysiological parameters. A) Examples of wave life spans in control, $I_{K}$ blockade, PVI, and PVI $+I_{K}$ blockade simulations with severe fibrosis (the slope of the fitted red line indicates the wave generation rate). B) Average number of waves per cycle. C) Average wave sizes per cycle. D) Wave generation rate. E) Wave lifespan median. 
Table 1. Atrial fibrillation cycle length (AFCL) and Excitable gap (EG) in simulation groups 501 with different degrees of fibrosis.

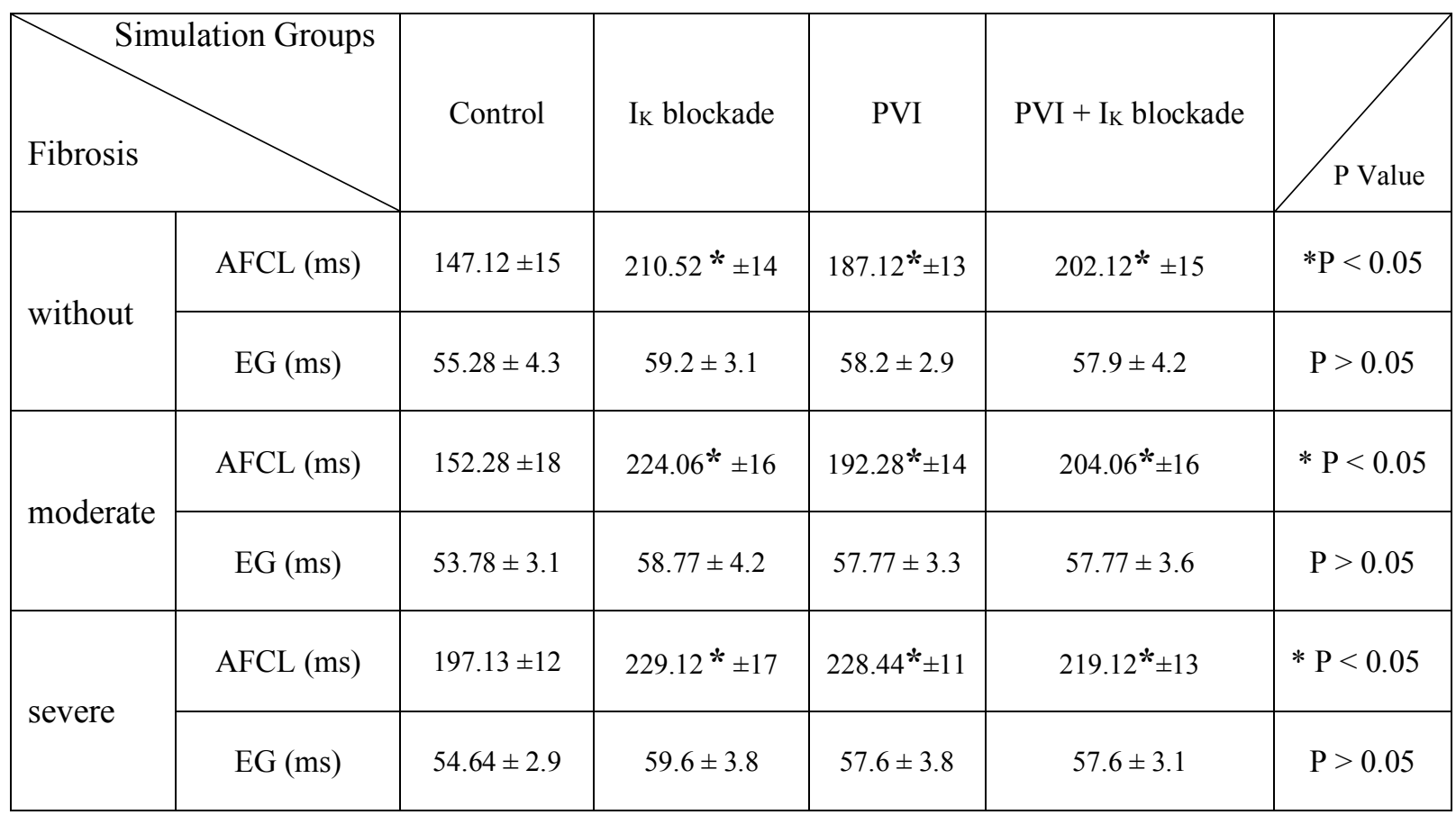


Figure 1

A

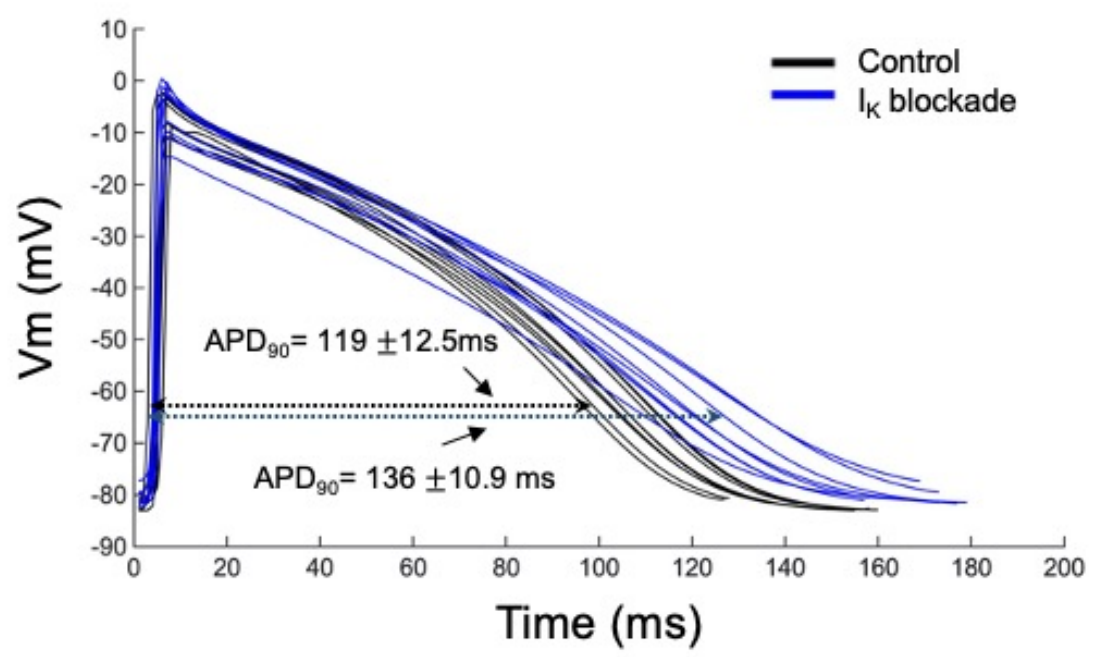

B

Pacing interval

C

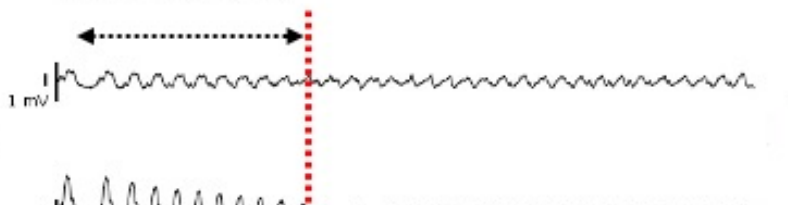

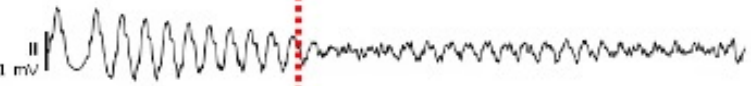

\section{Pacing interval}

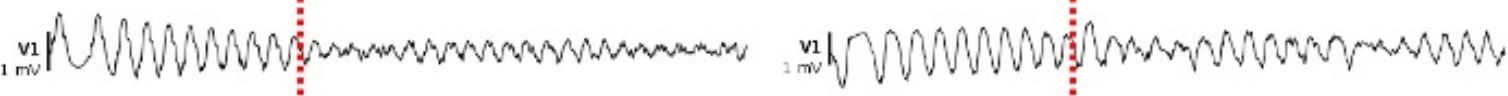

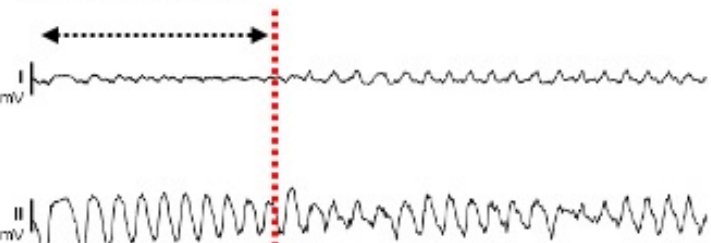

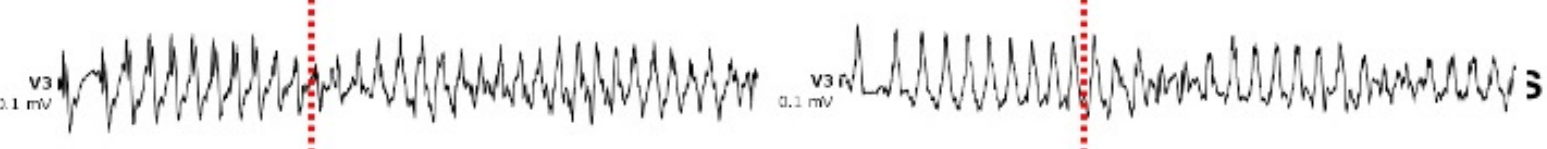

0

Time

$5 s$

0

Time 
Figure 2

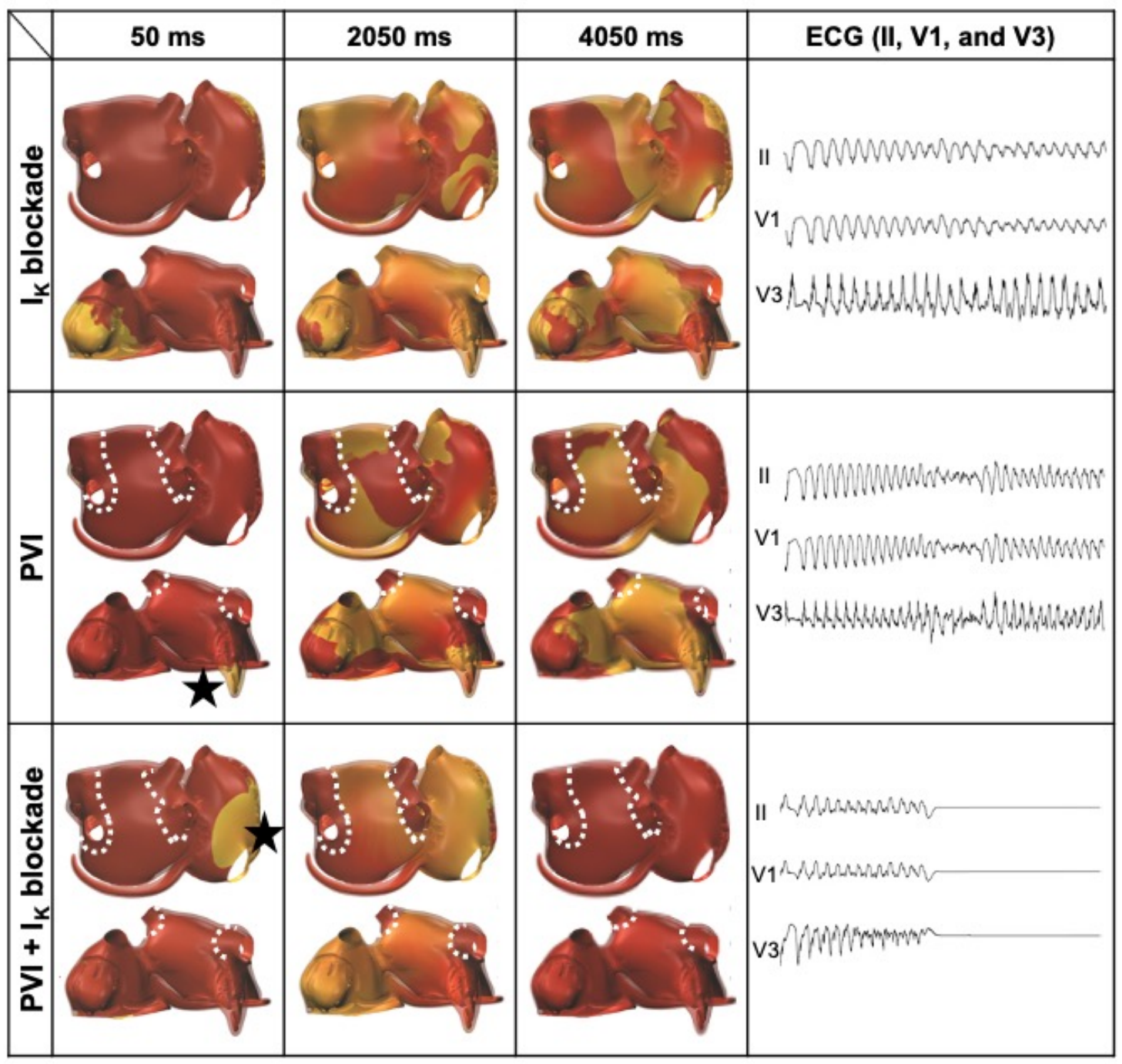


Figure 3

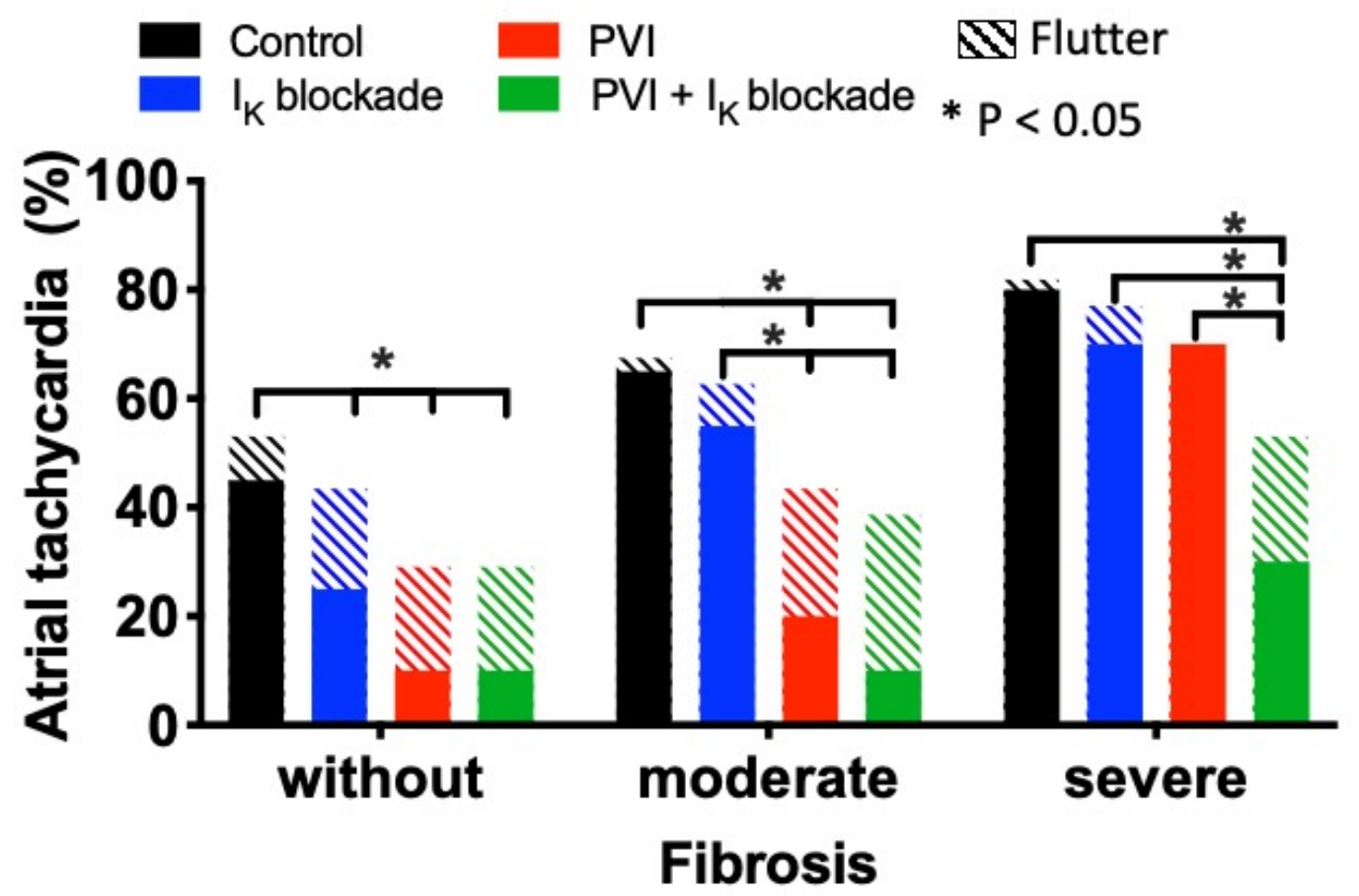


Figure 4

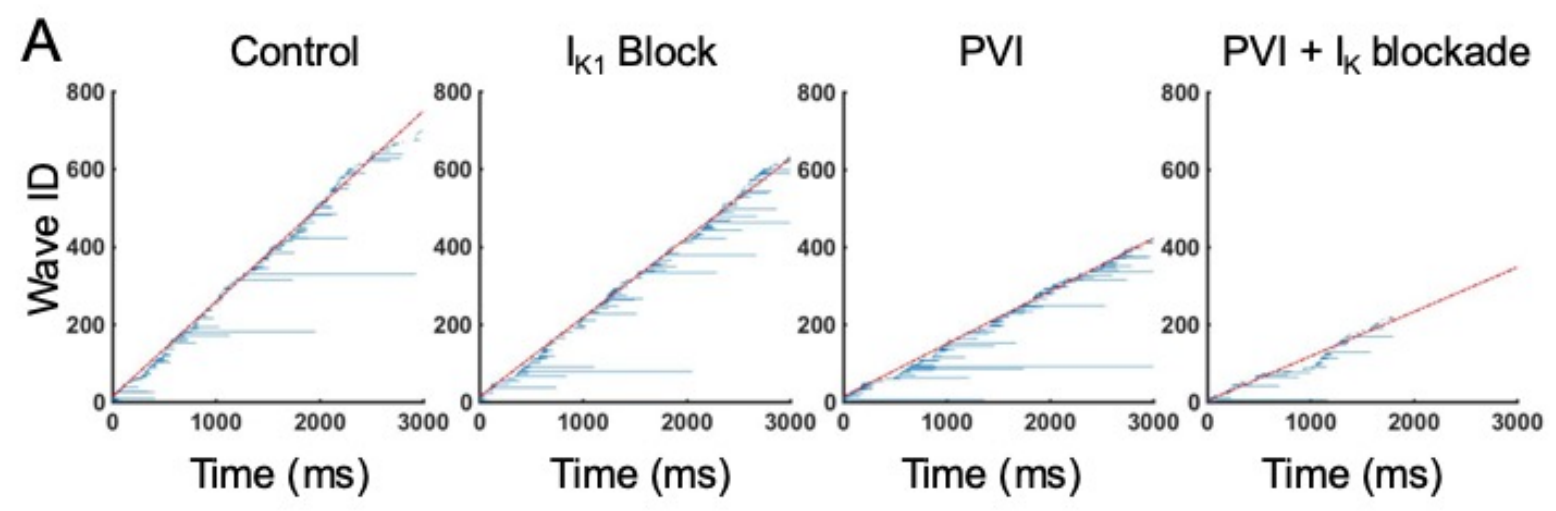

B

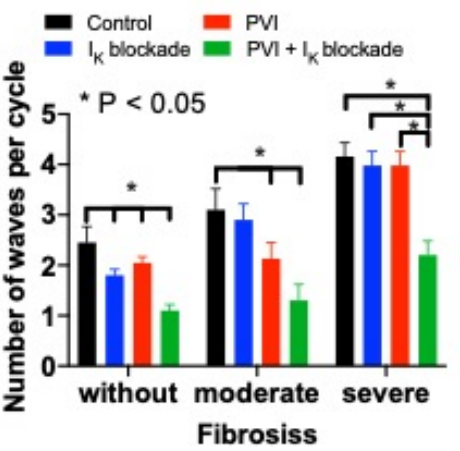

C

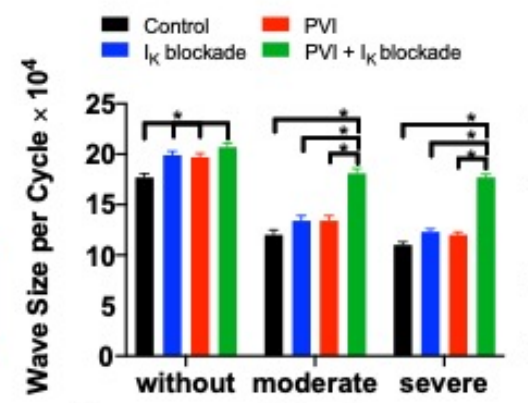

E
D

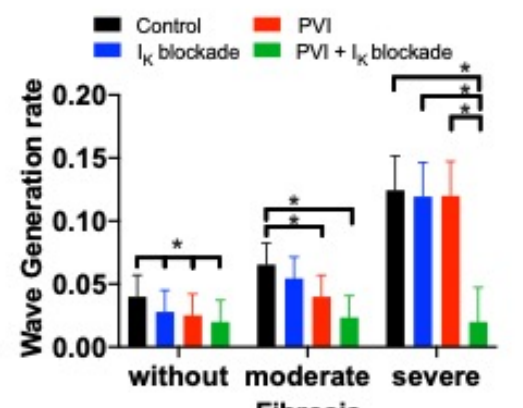

Fibrosis

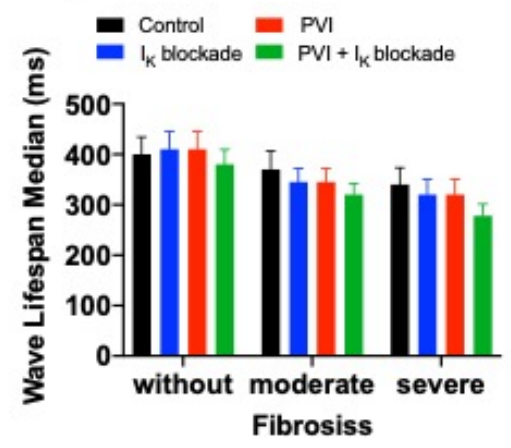

